\title{
Introduction au numéro thématique
}

Les structures industrielles sont le plus souvent des assemblages de composants de propriétés mécaniques différentes. Le choix du mode d'assemblage est guidé par des considérations techniques comme le poids, l'encombrement, la compatibilité des matériaux, la résistance de la structure... associées à des considérations économiques comme le coût. La prise en compte de l'assemblage dans un calcul de structure pose encore de nombreux problèmes aux industriels. En effet, bien que le réel « ignore » les assemblages, ceux-ci constituent des zones d'hétérogénéités et/ou de discontinuités avec de forts gradients de microstructures et de sollicitations, où se localiseront des problèmes susceptibles d'avoir de lourdes conséquences sur la fiabilité mécanique des structures. L'étude de la tenue des assemblages nécessite une approche pluridisciplinaire, multi-échelle, et la prise en compte des assemblages dans un calcul de structure nécessite des calculs fortement couplés entre la thermique, la métallurgie, la chimie, la mécanique... Un certain nombre de travaux de recherche sont développés depuis quelques années autour de la mécanique des assemblages. Le colloque MECAMAT qui s'est tenu à Aussois en janvier 2003, intitulé «Assemblages : des matériaux à la structure », se proposait de faire le point sur l'état de l'art de la prise en compte, dans un calcul de structure, des différents problèmes mécaniques soulevés par les assemblages, depuis leur élaboration jusqu'à leur utilisation. Le colloque traitait des joints soudés, des joints collés, des assemblages par contact (rivetage, boulonnage, vissage), mais aussi d'exemples d'assemblages multi-matériaux plus spécifiques. Des développements de techniques de modélisation spécifiques, nécessaires aux calculs des structures assemblées, étaient également présentés. Le présent numéro thématique de la revue Mécanique \& Industries se propose d'offrir un panel aussi large et représentatif que possible des travaux présentés à ce colloque.

L'équipe organisatrice :

Véronique AUBIN, Suzanne DEGALLAIX, Yannick DESPLANQUES, Marie-Claire TANGHE 